\title{
Valoración del Impacto funcional producido en participantes de taller laboral protegido
}

Labbé Matus, Elisa (1); Valdés González, Carola (2); Lûer Solorza, Katherin(3); et al.

(1)Terapeuta Ocupacional, Clínica Los Coihues,(2)(3)Licenciada Ciencias de la Ocupación Humana, Terapeuta Ocupacional,

Contacto > > Clínica Los Coihues, Av. Laguna Sur 6561, Estación Central, Fono: 4657900, elabbe@loscoihues.cl; cvaldes@loscoihues.cl; kluer@loscoihues.cl

Referencia > > Labbé Matus, Elisa; Valdés González, Carola ; Lûer Solorza, Katherin; et al." "Valoración del I mpacto funcional producido en participantes de taller laboral protegido". Revista Chilena de Terapia Ocupacional. №5. Noviembre 2005.

\section{- Abstract}

The main goal of this study is to demonstrate that maintaining an active work life has a determining impact on people's functional tasks. We also considered important to objectively assess the benefits of programs developed for disabled adults. We propose the use of the Functional Independence Assessment (FIA) to fulfil these goals.

This instrument was administered to 14 inpatients, $80 \%$ of which had suffered disabling traumatic brain injury, and $20 \%$ with spinal cord injury, all of whom have had attended the sheltered workshop of Clínica Los Coihues during a two year period. Their ages ranged between 29 and 74 years old, with an average of 56 years, and their average time of hospitalisation was of 8 years.

The FIA considers five performance areas and abilities: motor, social, cognitive, activities of daily living, and work.

We observed achievements in all of these areas, specially in the social and work aspects, although all areas were facilitated. Patients demonstrated high levels of personal satisfaction as well as direct benefits in their interpersonal relationships and mood.

It is important to emphasize a sense of

\section{Resumen}

El objetivo principal del estudio es demostrar que el área de desempeño del trabajo tiene una influencia determinante en el quehacer funcional de las personas. Por otra parte es importante poder evaluar y objetivar los logros de los programas de acción dirigidos hacia personas discapacitadas. Para ello se propone el siguiente instrumento de evaluación: la Pauta de Independencia Funcional (PIF) ${ }^{(*)}$.

Este instrumento fue aplicado a una población de 14 pacientes que han sufrido un accidente de trabajo y/o trayecto y cuya condición actual es de institucionalización, De estos el $80 \%$ secuelados de TEC y $20 \%$ lesionados medulares que asisten al Taller Laboral Protegido durante un período de 2 años, rango de edad entre 29 y 74 años (promedio 56 años y tiempo de institucionalización de 8 años promedio).

La evaluación PIF consideró 5 áreas en su desempeño y habilidades: motora, social, cognitiva, actividades de la vida diaria y laboral.

En todas ellas se observaron logros, principalmente en el área social y laboral, siendo facilitadas también las otras áreas. Los pacientes muestran altos niveles de 
belonging to a group, a greater identity in social roles, and an increase in significant verbal and non verbal expression, which represent volitional indicators that are inherent to the human being as a person. These factors are demonstrated in the FIA satisfacción personal y beneficios directos en relaciones interpersonales, ánimo.

Es importante destacar el sentido de pertenencia al grupo, mayor identidad en roles sociales y un discreto cambio en la expresión verbal y no verbal que manifiesta indicadores volicionales inherentes al ser humano como persona, estos factores se reflejan en la pauta PIF.

$\stackrel{*}{*}$ Registro Propiedad Intelectual $N^{\circ} 149.076$

\section{" Introducción "}

Se ha definido ocupación como "la variedad de actividades significativas personal y culturalmente en las que participan los seres humanos". Se debe considerar también que las personas organizan su rutina diaria mediante la planificación y participación en las ocupaciones.

Las ocupaciones pueden explicarse mediante los sustratos de la estructura, función, significado y contexto. La estructura incluye los productos, actividades, propiedades y las relaciones con el tiempo. Las funciones incluyen los procesos y la experiencia, en tanto el significado incorpora las motivaciones y la satisfacción personal. El contexto nos enmarca en las definiciones sociales y las perspectivas históricas en que vivimos. Desde esta base podemos definir la importancia del trabajo para la persona.

El trabajo generalmente se define en función de ganarse la vida, en empleo remunerado o tareas productivas. Las características que distinguen al trabajo son:

1. recompensa extrínseca,

2. derechos y obligaciones formales,

3. desempeño en límites de tiempo,

4. tareas determinadas $y$

5. predecible $^{(1)}$.

En el año 2002, en Clínica Los Coihues se crea un Taller Protegido de carácter laboral dirigido a pacientes de ACHS que tienen la condición de institucionalizados. El Taller funciona al interior de la Clínica Los Coihues. Se entiende por Paciente institucionalizado ${ }^{(2)}$; " aquellos pacientes que en razón de las secuelas del accidente de trabajo, trayecto o enfermedad profesional han quedado con un severo daño neurológico o mental que, por la naturaleza de su déficit, la complejidad de su manejo o las condiciones sociales de su medio, deben ser internados en centros especializados o requieren de ayuda de terceros en domicilio, definiendo para ellos distintas modalidades de atención que contempla el Programa de Pacientes Institucionalizados PPI".

El Taller esta dirigido a aquellos pacientes institucionalizados cuya reinserción laboral se ha observado limitada por sus condiciones físicas/ mentales al no responder a las exigencias actuales del mercado. Este Taller estimula las potencialidades remantes y capacidades resilientes de la persona, que favorezca la realización de una vida útil y con sentido de vida a pesar de sus secuelas y condiciones 
físicas, que va más allá de la reinserción al mundo laboral competitivo.

Para conocer el impacto que ha tenido el Taller en la capacidad funcional de la persona, se realizó un estudio que permita evaluar los logros y grado de autonomía alcanzados en los pacientes.

Al trabajar con estos pacientes se pueden observar logros significativos en el área social y desempeño funcional, sin embargo no se contaba con algún instrumento de evaluación que permitiera objetivar dichos logros y resultados. Surge entonces, después de una amplia revisión bibliográfica, la elaboración de la Pauta de Independencia Funcional (PIF) ${ }^{(*)}$ como una propuesta para las instituciones que trabajan en rehabilitación. La pauta contempla las siguientes áreas: motora, social, cognitiva, actividades de la vida diaria y laboral.

En el área motora se consideran los aspectos de desplazamientos (marcha o uso de silla de ruedas) y transferencias para que la persona pueda acceder a cualquier actividad. Requiere trasladarse en forma autónoma, favoreciendo la independencia en el manejo interno en un espacio rutinario. Se entiende por manejo interno que la persona pueda desplazarse al interior de un lugar conocido, con marcha autónoma o con ayudas técnicos.

Es fundamental en el área social durante el desempeño diario tener una condición emocional estable, desarrollar relaciones interpersonales y de comunicación adecuadas al contexto físico social, y ser capaz de ocupar el tiempo libre en actividades de interés.

En el área cognitiva para lograr realizar las tareas indicadas la persona debe: comprender instrucciones, expresar sus necesidades, resolver problemas básicos, retener y ejecutar la información.

Las actividades de la vida diaria permiten el autocuidado, mejorando la imagen que el individuo tiene de sí mismo y en su autonomía en cuanto a aspectos personales (alimentación, baño, vestuario, y otras) y sociales (manejo interno y externo).

El manejo externo es la capacidad de la persona de trasladarse en espacios públicos, uso de locomoción colectiva, incluyendo habilidades cognitivas como manejo de dinero atención, orientación tempo-espacial, comprensión, expresión y/o lenguaje verbal, memoria.

Todas estas áreas en su conjunto permiten que la persona pueda lograr autonomía en el diario vivir y a la vez tenga alternativas en el aspecto laboral, donde se miden los hábitos laborales, iniciativa, adaptabilidad, motivación y desempeño de roles, requisitos indispensables para la adquisición de roles laborales y lograr permanecer en un puesto de trabajo protegido ${ }^{3}$.

(*) $\quad$ Registro de Propiedad Intelectual No 149.076

\section{- Objetivos "}

\section{Objetivo General:}

1. Objetivar que la persona con discapacidad que participa en un taller laboral obtiene logros en las áreas de desempeño.

\section{Objetivos Específicos:}


1. Evaluar el impacto del Taller Protegido en las personas con discapacidad severa institucionalizadas.

2. Identificar indicadores de funcionalidad en programas de acción sobre discapacitados

\section{" Material y Método}

Se estudian 14 pacientes de sexo masculino, entre 29 y 74, con una edad promedio de 56 años, portadores de secuelas neurológicas graves como consecuencias de accidentes del trabajo, promedio de institucionalización de 8 años (Tabla $N^{\circ} 1$ ). Todos ellos asisten regularmente al Taller Protegido, durante 2 años un 93\% (13 personas) y 1 año y medio el $7 \%$ (1 persona). Se realizó un estudio exploratorio, descriptivo y prospectivo, con enfoque cuantitativo.

\section{Tabla N¹}

\begin{tabular}{|l|c|c|c|}
\hline Pacientes & $\begin{array}{c}\text { Edad a la } \\
\text { fecha } \\
\text { de evaluación }\end{array}$ & Diagnóstico & $\begin{array}{c}\text { Tiempo de } \\
\text { participación } \\
\text { en Taller Laboral }\end{array}$ \\
\hline Paciente 1L. A. & 46 años & Secuelado TEC & 2 años \\
\hline $\begin{array}{l}\text { Paciente 2 J. M. } \\
\text { A }\end{array}$ & 32 años & Secuelado TEC & 2 años \\
\hline Paciente 3 J. B. & 35 años & $\begin{array}{c}\text { Lesionado } \\
\text { Medular }\end{array}$ & 2 años \\
\hline Paciente 4 P M. & 55 años & Secuelado TEC & 2 años \\
\hline Paciente 5 P. P. & 49 años & Secuelado TEC & 2 años \\
\hline Paciente 6 M .N. & 58 años & Secuelado TEC & 2 años \\
\hline Paciente 7 U. O. & 55 años & Secuelado TEC & 2 años \\
\hline Paciente 8 J. V. & 65 años & $\begin{array}{c}\text { Lesionado } \\
\text { Medular }\end{array}$ & 2 años \\
\hline Paciente 9 J. V. & 55 años & Secuelado TEC & 2 años \\
\hline Paciente 10 J. S. & 32 años & Secuelado TEC & 2 años \\
\hline Paciente M. R. & 71 años & Secuelado TEC & 2 años \\
\hline Paciente 12 R. V. & 62 años & Secuelado TEC & 2 años \\
\hline Paciente 13 J. P. & 26 años & $\begin{array}{c}\text { Lesionado } \\
\text { Medular }\end{array}$ \\
\hline Paciente14 L. R. & 64 años & Secuelado TEC & 1 año \\
\hline \hline
\end{tabular}

Las respuestas fueron evaluadas a nivel de la medición de capacidad funcional.

Se aplicó el instrumento a cada persona: Pauta de Independencia Funcional (PIF).

La Pauta de Independencia Funcional (PIF) es aplicada al ingreso del Taller, a un año de evolución (2003) y su condición al momento de la re-evaluación (Julio 2004).

La Pauta Independencia Funcional (PIF) está inspirado en las pautas FIM $^{(a)}$ y FAM ${ }^{(b)}$, instrumento que fue ideado en el contexto del taller laboral, sobre la base de la experiencia y necesidades de los 
pacientes del taller. Esta pauta considera 5 aspectos de los participantes en su desempeño tales como: aspecto motor, social, cognitivo, actividades de la vida diaria y laboral. El puntaje para cada ítem se valora en escala de 1 a 7 , siendo el valor 1 para las personas que requieren máxima asistencia y el valor 7 para aquellas que son autónomas o auto gestionadoras. La puntuación máxima para esta evaluación es 126 y la mínima 18.

Se analizaron estadísticamente los datos obtenidos en el PIF, donde el valor "Tcrit" que mide el nivel de significación de los diferentes aspectos medidos debe ser igual o superior a 2.16.

(a) FIM: Functional Independence Measure
(b) $\quad$ FAM:Functional Assessment Measurement

\section{Resultados}

Pauta Independencia Funcional (PIF)

- Evolución del PIF

Se efectuó el seguimiento de la evolución de los 14 pacientes del estudio a través del PIF, 13 de ellos experimentaron una evolución favorable, medido a través del incremento en la puntuación. En la Tabla $\mathrm{N}^{0} 2$ se presenta el cambio porcentual experimentado por cada uno de los pacientes comparando el puntaje de ingreso (2002), a un año (2003) y el obtenido a los dos años (2004) de evaluación. En el transcurso del tiempo se aprecia un rango de incremento variable en cada uno de los pacientes. La tendencia es que ha mejorado el puntaje obtenido en independencia funcional, siendo en promedio la evolución favorable de un $29 \%$. Se observaron logros importantes en todas las áreas de evaluación (motora, social, actividades de la vida diaria, cognitiva y laboral).

Tabla №1

\begin{tabular}{|l|c|l|c|}
\hline Pacientes & $\begin{array}{c}\text { Edad a la } \\
\text { fecha } \\
\text { de evaluación }\end{array}$ & Diagnóstico & $\begin{array}{c}\text { Tiempo de } \\
\text { participación } \\
\text { en Taller Laboral }\end{array}$ \\
\hline Paciente 1L. A. & 46 años & Secuelado TEC & 2 años \\
\hline $\begin{array}{l}\text { Paciente 2 J. M. } \\
\text { A }\end{array}$ & 32 años & Secuelado TEC & 2 años \\
\hline Paciente 3 J. B. & 35 años & $\begin{array}{c}\text { Lesionado } \\
\text { Medular }\end{array}$ & 2 años \\
\hline Paciente 4 P M. & 55 años & Secuelado TEC & 2 años \\
\hline Paciente 5 P. P. & 49 años & Secuelado TEC & 2 años \\
\hline Paciente 6 M .N. & 58 años & Secuelado TEC & 2 años \\
\hline Paciente 7 U. O. & 55 años & Secuelado TEC & 2 años \\
\hline Paciente 8 J. V. & 65 años & $\begin{array}{c}\text { Lesionado } \\
\text { Medular }\end{array}$ & 2 años \\
\hline Paciente 9 J. V. & 55 años & Secuelado TEC & 2 años \\
\hline
\end{tabular}




\begin{tabular}{|l|c|c|c|}
\hline Paciente 10 J. S. & 32 años & Secuelado TEC & 2 años \\
\hline Paciente M. R. & 71 años & Secuelado TEC & 2 años \\
\hline Paciente 12 R. V. & 62 años & Secuelado TEC & 2 años \\
\hline Paciente 13 J. P. & 26 años & $\begin{array}{c}\text { Lesionado } \\
\text { Medular }\end{array}$ & 2 años \\
\hline Paciente14 L. R. & 64 años & Secuelado TEC & 1 año \\
\hline
\end{tabular}

Tabla No2

\begin{tabular}{|l|c|c|c|c|}
\hline \multicolumn{5}{|c|}{ Resultados PIF Total (Puntaje) } \\
\hline Pacientes & Jul-02 & Jul-03 & Jul-04 & $\begin{array}{c}\text { Cambio \% } \\
\text { a partir del ingreso. }\end{array}$ \\
\hline Paciente 1 & 74 & 72 & 99 & $34 \%$ \\
\hline Paciente 2 & 63 & 86 & 96 & $52 \%$ \\
\hline Paciente 3 & 23 & 34 & 38 & $65 \%$ \\
\hline Paciente 4 & 103 & 106 & 109 & $6 \%$ \\
\hline Paciente 5 & 89 & 97 & 103 & $16 \%$ \\
\hline Paciente 6 & 57 & 66 & 72 & $26 \%$ \\
\hline Paciente 7 & 77 & 87 & 88 & $14 \%$ \\
\hline Paciente 8 & 102 & 104 & 107 & $5 \%$ \\
\hline Paciente 9 & 46 & 59 & 69 & $-24 \% *$ \\
\hline Paciente 10 & 58 & 45 & 44 & $30 \%$ \\
\hline Paciente 11 & 43 & 51 & 56 & $78 \%$ \\
\hline Paciente 12 & 40 & 64 & 71 & $12 \%$ \\
\hline Paciente 13 & 107 & 115 & 120 & $40 \%$ \\
\hline Paciente 14 & 0 & 35 & 58 & \\
\hline * Paciente portador de patología degenerativa asociada. \\
\hline
\end{tabular}

- Área Motora.

Los pacientes fueron evaluados en función del grado de dependencia en desplazamiento y transferencias. Los puntajes para cada ítem se valoran en escala de 1 a 7 , siendo el valor 1 para las personas que requieren máxima asistencia.En la Tabla $N^{\circ} 3$ se muestran los resultados obtenidos en el año 2002 y año 2004 del total de los pacientes, las diferencias promedios y desviación estándar entre ambos años. Se observa evolución favorable las áreas de desplazamiento y transferencia, con una DESV STD de 2.2 y 2.5 respectivamente.

Tabla N³

\begin{tabular}{|l||c|c|}
\hline \multicolumn{1}{|c||}{} & \multicolumn{2}{c|}{ AREA MOTORA } \\
\hline Resultados & Desplazamiento & Transferencia \\
\hline \hline Año 2002 & & \\
\hline Promedio & 4,2 & 4,2 \\
\hline \hline DESV STD & 2,3 & 2,4 \\
\hline
\end{tabular}




\begin{tabular}{|l|c|c|}
\hline Año 2004 & & \\
\hline Promedio & 4,8 & 4,6 \\
\hline DESV STD & 2,1 & 2,5 \\
\hline $\begin{array}{l}\text { Análisis de diferencias * 2002 } \\
\text { I2004 }\end{array}$ & 0.6 & 0.4 \\
\hline $\begin{array}{l}\text { Promedio de las diferencias } \\
\text { individuales }\end{array}$ & 1.5 & 1.5 \\
\hline DESV STD & 1.42 & 0.89 \\
\hline Valor de t (t crit = 2.16 ) & No Significativo & No Significativo \\
\hline \hline Nivel significación & & \\
\hline $\begin{array}{l}\text { *Análisis para diferencia de variables continuas en muestras pareadas (N=14) } \\
\text { prueba t pareada. El valor “Tcrit"quemide el nivel de significación de los } \\
\text { diferentes aspectos medidos debe ser igual o superior a 2.16. }\end{array}$ \\
\hline
\end{tabular}

- Área Social.

Esta área considera para su medición la interacción social, las condiciones emocionales y actividades de tiempo libre. En ellas destacan las destrezas necesarias para participar con otros en situaciones sociales y terapéuticas. Se relaciona con el sentido de pertenencia al grupo, mayor identidad en roles sociales, la expresión verbal y no verbal que manifiesta indicadores volicionales inherentes al ser humano. Se distinguen dos situaciones a las que puede verse enfrentado la paciente, aquella en que la persona interactúa sin la asistencia de un tercero o bien en la cual requiere de la asistencia de otro para su desempeño.

En esta área y sus componentes, los pacientes mostraron una evolución positiva estadísticamente significativa en el transcurso de las mediciones.

Tabla N 4

\begin{tabular}{|c|c|c|c|}
\hline \multirow[t]{2}{*}{ Resultados } & \multicolumn{3}{|c|}{ AREA SOCIAL } \\
\hline & $\begin{array}{l}\text { Interacción } \\
\text { Social }\end{array}$ & $\begin{array}{l}\text { Condición } \\
\text { Emocional }\end{array}$ & $\begin{array}{l}\text { Actv. Tiempo } \\
\text { Libre }\end{array}$ \\
\hline \multicolumn{4}{|l|}{ Año 2002} \\
\hline Promedio & 3,5 & 3,1 & 3.1 \\
\hline DESV STD & 2,0 & 1,4 & 1.6 \\
\hline \multicolumn{4}{|l|}{ Año 2004} \\
\hline Promedio & 4,8 & 4,3 & 3.4 \\
\hline DESV STD & 1,7 & 1,6 & 1.9 \\
\hline \multicolumn{4}{|l|}{$\begin{array}{l}\text { Análisis de diferencias * } \\
2002 \text { /2004 }\end{array}$} \\
\hline $\begin{array}{l}\text { Promedio de las } \\
\text { diferencias individuales }\end{array}$ & 1.3 & 1.3 & 1.1 \\
\hline DESV STD & & 1.3 & \\
\hline- & 1.5 & 1.5 & 1.5 \\
\hline Valor de $\mathrm{t}(\mathrm{t}$ crit $=2.16)$ & 3.23 & 3.23 & 2.83 \\
\hline Nivel significación & $P<0.05$ & $P<0.05$ & $P<0.05$ \\
\hline
\end{tabular}


* Análisis para diferencia de variables continuas en muestras pareadas $(\mathrm{N}=14)$ prueba t pareada. El valor "Tcrit" que mide el nivel de significación de los diferentes aspectos medidos debe ser igual o superior a 2.16.

\section{- Área Cognitiva.}

Esta área considera: comprensión de la comunicación auditiva o visual; expresión verbal o no verbal del lenguaje; resolución de problemas en la vida diaria; memoria.

Estos aspectos implican que la persona adquiera y desarrolle habilidades para la toma de decisiones, razonables, seguras y complejas acorde a su nivel de comprensión, la información que se genera en instancias de intercambio grupal y la comprensión de información, instrucciones y manifestación de necesidades.

En esta área, los pacientes mostraron una evolución significativa en tres de sus componentes, destacando el nivel de comprensión de las instrucciones dadas y la resolución de problemas. Al igual que áreas anteriores se pueden presentar dos situaciones a las que puede enfrentarse el paciente, aquella en que la persona interactúa sin la asistencia de un tercero o bien en la cual requiere de la asistencia de otro para su desempeño. (Tabla $N^{\circ} 5$ ).

Tabla N 5

\begin{tabular}{|c|c|c|c|c|}
\hline \multirow[t]{2}{*}{ Resultados } & \multicolumn{4}{|c|}{ AREA COGNITIVA } \\
\hline & Comprensión & Expresión & $\begin{array}{l}\text { Resolución de } \\
\text { problemas }\end{array}$ & Memoria \\
\hline \multicolumn{5}{|l|}{ Año 2002} \\
\hline Promedio & 4.2 & 4.0 & 2.5 & 4.1 \\
\hline DESV STD & 1.7 & 2.2 & 1.8 & 2.3 \\
\hline \multicolumn{5}{|l|}{ Año 2004} \\
\hline Promedio & 4.9 & 4.6 & 3.5 & 4.6 \\
\hline DESV STD & 1.4 & 1.9 & 2.2 & 2.1 \\
\hline \multicolumn{5}{|l|}{$\begin{array}{l}\text { Análisis de } \\
\text { diferencias * } 2002 \\
\text { I2004 }\end{array}$} \\
\hline $\begin{array}{l}\text { Promedio de las } \\
\text { diferencias } \\
\text { individuales }\end{array}$ & 07 & 0.6 & 1.0 & 0.5 \\
\hline DESV STD & 0.6 & 0.9 & 1.0 & 0.9 \\
\hline $\begin{array}{l}\text { Valor de } \mathrm{t} \text { (t crit = } \\
2.16 \text { ) }\end{array}$ & 4.37 & 2.28 & 3.89 & 1.99 \\
\hline Nivel significación & $P<0.05$ & $P<0.05$ & $P<0.05$ & $\begin{array}{c}\text { No } \\
\text { significativo }\end{array}$ \\
\hline $\begin{array}{l}\text { * Análisis para difer } \\
\text { prueba t pareada. } \\
\text { diferentes aspectos }\end{array}$ & $\begin{array}{l}\text { ia de varial } \\
\text { lor "Tcrit" q } \\
\text { didos debe }\end{array}$ & $\begin{array}{l}\text { ontinuas } \\
\text { ide el nis } \\
\text { igual o st }\end{array}$ & $\begin{array}{l}\text { muestras pare } \\
\text { de significación } \\
\text { ior a } 2.16 \text {. }\end{array}$ & $\begin{array}{l}\text { las }(N=14) \\
\text { e los }\end{array}$ \\
\hline
\end{tabular}


- Actividades de la Vida Diaria.

Esta área contempla higiene personal, manejo de necesidades básicas, desplazamiento interno (ambiente protegido) y externo (comunidad).

Se observa una evolución importante y significativa en la expresión y manejo que los pacientes hacen de sus necesidades básicas, higiene personal y manejo externo. En manejo interno hubo avances pero no significativos.

Tabla N6

\begin{tabular}{|c|c|c|c|c|}
\hline & \multicolumn{4}{|c|}{ ACTIVIDADES DE LA VIDA DIARIA } \\
\hline & Higiene & $\begin{array}{l}\text { Manejo } \\
\text { Necesidades } \\
\text { Básicas }\end{array}$ & $\begin{array}{l}\text { Manejo } \\
\text { Interno }\end{array}$ & $\begin{array}{l}\text { Manejo } \\
\text { Externo }\end{array}$ \\
\hline \multicolumn{5}{|l|}{ Año 2002} \\
\hline Promedio & 2.7 & 3.5 & 4.1 & 3.1 \\
\hline DESV STD & 2.0 & 2.0 & 2.1 & 1.9 \\
\hline \multicolumn{5}{|l|}{ Año 2004} \\
\hline Promedio & 3.5 & 4.5 & 4.8 & 4.0 \\
\hline DESV STD & 2.1 & 1.8 & 1.9 & 2.2 \\
\hline \multicolumn{5}{|l|}{$\begin{array}{l}\text { Análisis de } \\
\text { diferencias * } 2002 \\
12004\end{array}$} \\
\hline $\begin{array}{l}\text { Promedio de las } \\
\text { diferencias } \\
\text { individuales }\end{array}$ & 0.8 & 1.0 & 0.6 & 0.9 \\
\hline DESV STD & 1.1 & 1.0 & 1.6 & 1.4 \\
\hline $\begin{array}{l}\text { Valor de } \mathrm{t}(\mathrm{t} \text { crit }= \\
2.16)\end{array}$ & 2.62 & 3.61 & 1.50 & 2.37 \\
\hline Nivel significación & $P<0.05$ & $P<0.05$ & $\begin{array}{c}\text { No } \\
\text { significativo }\end{array}$ & $P<0.05$ \\
\hline \multicolumn{5}{|c|}{$\begin{array}{l}\text { * Análisis para diferencia de variables continuas en muestras pareadas (N=14) } \\
\text { prueba t pareada. El valor "Tcrit" que mide el nivel de significación de los } \\
\text { diferentes aspectos medidos debe ser igual o superior a } 2.16 \text {. }\end{array}$} \\
\hline
\end{tabular}

- Área Laboral.

Contempla la presencia de ciertas habilidades que se requieren para desarrollar una tarea en forma más autónoma, que impliquen un mejor desempeño laboral, aún cuando se trate de un ambiente protegido, relacionado con la productividad y la adaptabilidad al sistema.

La evaluación y evolución de estos aspectos resultaron altamente significativos, destacando roles sociales, hábitos laborales y adaptabilidad. Sin embargo estos resultados deben ser relacionados con la capacidad de independencia, como por ejemplo el desplazamiento, para obtener un resultado en lo laboral, lo que significa que el paciente igualmente debe permanecer en un medio protegido. 
Tabla N7

\begin{tabular}{|c|c|c|c|c|c|}
\hline \multirow[t]{2}{*}{ Resultados } & \multicolumn{5}{|c|}{ AREA LABORAL } \\
\hline & Iniciativa & $\begin{array}{l}\text { Hábitos } \\
\text { Laborales }\end{array}$ & $\begin{array}{l}\text { Adaptabi- } \\
\text { lidad }\end{array}$ & $\begin{array}{l}\text { Motiva- } \\
\text { ción }\end{array}$ & $\begin{array}{c}\text { Roles } \\
\text { Sociales }\end{array}$ \\
\hline \multicolumn{6}{|l|}{ Año 2002} \\
\hline Promedio & 3.4 & 3.9 & 3.8 & 4.7 & 2.8 \\
\hline DESV STD & 2.0 & 2.0 & 1.8 & 2.2 & 1.8 \\
\hline \multicolumn{6}{|l|}{ Año 2004} \\
\hline Promedio & 4.3 & 5.1 & 4.6 & 5.5 & 3.5 \\
\hline DESV STD & 2.0 & 1.5 & 1.7 & 1.5 & 1.7 \\
\hline \multicolumn{6}{|l|}{\begin{tabular}{|l|} 
Análisis de \\
diferencias * 2002 \\
2004
\end{tabular}} \\
\hline $\begin{array}{l}\text { Promedio de las } \\
\text { diferencias } \\
\text { individuales }\end{array}$ & 0.9 & 1.2 & 0.8 & 0.8 & 0.7 \\
\hline DESV STD & 1.0 & 1.2 & 0.9 & 1.2 & 0.5 \\
\hline $\begin{array}{l}\text { Valor de } \mathrm{t} \text { ( } \mathrm{t} \text { crit }= \\
2.16 \text { ) }\end{array}$ & 3.12 & 3.82 & 3.29 & 2.47 & 5.70 \\
\hline Nivel significación & $P<0.05$ & $P<0.05$ & $P<0.05$ & $P<0.05$ & $P<0.05$ \\
\hline \multicolumn{6}{|c|}{$\begin{array}{l}\text { * Análisis para diferencia de variables continuas en muestras pareadas }(\mathrm{N}=14) \\
\text { prueba t pareada. El valor "Tcrit" que mide el nivel de significación de los } \\
\text { diferentes aspectos medidos debe ser igual o superior a } 2.16 \text {. }\end{array}$} \\
\hline
\end{tabular}

\section{" Discusión y Conclusiones "}

El estudio incorpora la necesidad de tener instrumentos de evaluación que permitan objetivar los cambios que se producen en las personas con discapacidad al tener una actividad significativa y organizadora de su rutina de vida; por lo cual se diseña y aplica un instrumento de evaluación y seguimiento, Pauta de Independencia Funcional (PIF) destinado a pacientes discapacitados que asisten a un Taller Protegido.

Las conclusiones principales de la aplicación del instrumento fueron las siguientes:

- Existe una alta valorización de los pacientes respecto a los beneficios que aporta el taller en su vida diaria, especialmente en los aspectos de relaciones interpersonales, y estabilidad del ánimo.

- Al aplicar la pauta de independencia funcional (PIF) se advierte que el taller logra avances importantes en las áreas motoras, sociales, cognitiva, actividades de la vida diaria y laboral. Esta evaluación nos permite mostrar avances objetivos y medibles en los logros de los pacientes en las diferentes áreas.

- En el área motora se logran avances en función del grado de dependencia en desplazamiento y transferencias, sin embargo estos no son del todo significativos y están estrictamente relacionados con la patología y secuelas que presenta el paciente, resultando ésta su mayor complejidad para tener un grado mayor de funcionalidad, autonomía y rendimiento laboral.

- En las áreas social y laboral, destaca el sentido de pertenencia al grupo, mayor identidad en 
roles sociales y un discreto cambio en la expresión verbal y no verbal que manifiesta indicadores volicionales inherentes al ser humano como persona.

- Impresiona la estabilidad alcanzada por los pacientes en cuanto al aspecto emocional, lo que se traduce una disminución de consultas médicas en el período de permanencia en el Taller.

- También es posible que en los resultados obtenidos estén influyendo otras acciones y actividades en que participan los pacientes y que estimulan la interacción social tales como salidas a la comunidad, oración semanal, socioterapias, que favorecen la adecuación al medio social y físico que les permiten mantenerse orientados y más conectados.

- El área social y laboral, se ven favorecidas con la participación de los pacientes en el Taller, debido a que la forma de funcionar de éste, persigue justamente una influencia directa y fortalecer estos ámbitos de la ocupación humana; social y laboral, sin desconocer que son importantes también y se ven facilitados por el Taller las áreas motora, cognitiva y actividades de la vida diaria.

- La evaluación y evolución de estos aspectos resultan ser importantes y altamente significativos, destacando roles, hábitos laborales y adaptabilidad, sin embargo estos resultados deben ser relacionados con la capacidad de independencia por ejemplo en traslado de cada paciente para obtener un resultado en lo laboral, lo que significa que el paciente igualmente debe permanecer en un medio protegido. (F. Área laboral)

- En cuanto a otros logros en lo laboral, en el transcurso del tiempo de desarrollo del taller, dos pacientes fueron reinsertados laboralmente en un carácter protegido en un establecimiento comercial, sección bodega, finalizando la tarea y periodo convenido con éxito. Diez de los pacientes participantes al taller lo hacen en condición ambulatoria, 2 de estos se reintegran a sus familias, los otros 8 pacientes fueron derivados a un centro de menor complejidad y asisten diariamente al taller.

\section{- Referencias =}

(1) Willard y Spackman, Terapia Ocupacional, Ed. Panamericana, 8va. Edición, págs. 260, 261, 2001.

(2) Acevedo M, Barna R y Carrasco L. Características de las familias y exigencias de intervención familiar en discapacitados institucionalizados. Artículo original publicado Revista Chilena de Medicina Familiar Vol IV.Nº3 Diciembre 2003.

(3) http://www.mtas.es/insht/EncOIT/pdf/tomo1/17.pdf , (mayo, 2004).

\section{- Agradecimientos}

Agradecimientos: los autores expresan su agradecimiento a la Dra. Francisca Paravic, Médico Fisiatra Clínica Los Coihues, por apoyo técnico, al Dr. Gonzalo Lira por su apoyo en el análisis estadístico para la realización de este trabajo, y a nuestra colega; Anne Marie Bonnefoy por su colaboración en traducción 